\title{
Candida parapsilosis in the blood smear of an injection drug user
}

\section{Konstantinos Liapis MD}

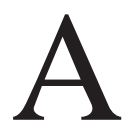
33-year-old man with a history of intravenous drug use presented to the emergency department with fever, rigors, cough and malaise of seven days' duration. He had been injecting heroin for five years and was known to be hepatitis-C positive. On physical examination, he appeared acutely ill with a fever of $39.5^{\circ} \mathrm{C}$, moderate respiratory distress (respiratory rate 26 breaths/min, oxygen saturation 94\%) and basilar rales over his right lung. He had a painful nodular skin lesion on his left arm corresponding to an injection site and track marks on both arms. His leukocyte count was 29.1 (normal 4.0-10.5) $\times 10^{\circ} / \mathrm{L}$, hemoglobin level was 120 (normal 130-175) g/L, platelet count was 83 (normal $140-450) \times 10^{\circ} / \mathrm{L}$, alanine transaminase level was 104 (normal 5-40) U/L and C-reactive protein level was 108.4 (normal < 10) mg/L. A peripheralblood smear showed extracellular and intracellular budding yeasts being phagocytized by neutrophils. The yeast forms consisted of pseudohyphae and blastospores (Figure 1; Appendix 1, available at www.cmaj.ca/cgi/content/full/cmaj.101947/DC1).

After gathering blood and urine cultures, we started the patient on liposomal amphotericin B. Computed tomography showed three nodular opacities affecting the patient's right lung and a small hypodense lesion in his left kidney. Transesophageal echocardiography and ophthalmologic evaluation were unremarkable. Two days later, Candida parapsilosis was isolated in blood and urine cultures, and serologic tests came back positive for antibodies against the hepatitis $\mathrm{C}$ virus and negative for HIV. After 15 days of treatment with amphotericin B, our patient received a two-week course of fluconazole. One month later, he had no signs of residual or recurrent infection.

Injection drug users are at increased risk for blood-borne invasive bacterial and fungal infections. ${ }^{1}$ Deep candidiasis is the most frequent invasive fungal infection among this group, although its precise incidence is unknown. Candidiasis may be disseminated or confined to one organ, frequently presenting as endocarditis or endoph-

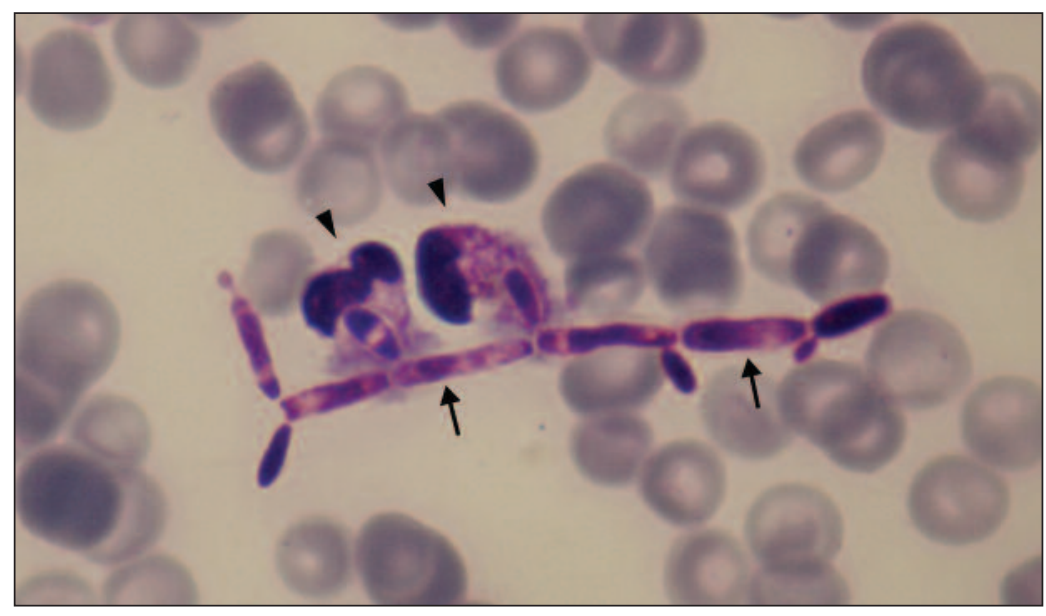

Figure 1: Peripheral-blood smear from an acutely ill 33-year-old man with a history of intravenous drug use, showing branched pseudohyphae (arrows) and two neutrophils in the process of phagocytizing fungal elements (arrowheads) (May-Grünwald, original magnification $\times 1000$ ).

thalmitis. ${ }^{1.2}$ Its pathogenesis involves mycotic contamination of drug paraphernalia (e.g., reused syringes) or contaminated drug solutions (e.g., through lemon juice used as an acidic solvent for brown heroin and crack cocaine). ${ }^{2}$

Candidemia in a peripheral-blood smear occurs only when fungal elements are present in high concentrations, in the range of $1-5 \times 10^{6}$ $\mathrm{CFU} / \mathrm{mL}$, and is therefore associated with highgrade infection and increased mortality. ${ }^{3.4}$ Because most candidemic episodes are characterized by much lower fungal loads, peripheral-blood smears play a limited role in early detection of disease. ${ }^{4}$

\section{References}

1. Edwards JE. Candida species. In: Mandell GL, Bennett JE, Dolin R, editors. Mandell, Douglas, and Bennett's principles and practice of infectious diseases. 6th ed. Philadelphia (PA): Elsevier Churchill Livingstone; 2005. p. 2938-57.

2. Rondon-Berrios H, Khilnani N, Trevejo-Nunez GJ, et al. Disseminated candidiasis in intravenous drug abusers: a distinctive syndrome. John Hopkins Advanced Studies in Medicine 2006;6:191-4.

3. Yera H, Poulain D, Lefebvre A, et al. Polymicrobial candidaemia revealed by peripheral blood smear and chromogenic medium. J Clin Pathol 2004;57:196-8.

4. Branda JA, Ferraro MJ, Kratz A. Sensitivity of peripheral blood smear review for the diagnosis of Candida fungemia. Arch Pathol Lab Med 2007;131:97-101.
Competing interests: None declared.

This article has been peer reviewed.

Affiliation: From the

Division of Internal

Medicine, Department of

Hematology, Sismanogleio

Hospital, Athens, Greece

Correspondence to: Dr. Konstantinos Liapis, kosliapis@hotmail.com

CMAJ 2011. DOI:10.1503 /cmaj.101947 